\title{
Kron 3: a fourth intermediate age cluster in the SMC with evidence of multiple populations ${ }^{\star}$
}

\author{
K. Hollyhead ${ }^{1} \dagger$, C. Lardo ${ }^{2}$, N. Kacharov ${ }^{3}$, N. Bastian ${ }^{4}$, M. Hilker ${ }^{5}$, M. Rejkuba $^{5,6}$, \\ A. Koch ${ }^{7,8}$ E. K. Grebel ${ }^{8}$ and I. Georgiev ${ }^{3}$ \\ 1 Department of Astronomy, Oscar Klein Centre, Stockholm University, AlbaNova, Stockholm SE-106 91, Sweden \\ ${ }^{2}$ Laboratoire d'astrophysique, Ecole Polytechnique Fèdèrale de Lausanne (EPFL), Observatoire de Sauverny, CH-1290 Versoix, Switzerland \\ ${ }^{3}$ Max-Planck-Institut für Astronomie, Königstuhl 17, D-69117 Heidelberg, Germany \\ ${ }^{4}$ Astrophysics Research Institute, Liverpool John Moores University, 146 Brownlow Hill, Liverpool L3 5RF, UK \\ ${ }^{5}$ European Southern Observatory, Karl-Schwarzschild-Straße 2, D-85748 Garching bei München, Germany \\ ${ }^{6}$ Excellence Cluster Universe, Boltzmannstr. 2, 85748, Garching, Germany \\ ${ }^{7}$ Department of Physics, Lancaster University, Lancaster LA1 4YB, UK \\ ${ }^{8}$ Astronomisches Rechen-Institut, Zentrum für Astronomie der Universität Heidelberg, Mönchhofstr. 12-14, 69120 Heidelberg, Germany
}

Accepted XXX. Received YYY; in original form ZZZ

\begin{abstract}
We present the results of a spectroscopic study of the intermediate age $(\approx 6.5 \mathrm{Gyr})$ massive cluster Kron 3 in the Small Magellanic Cloud. We measure CN and CH band strengths (at $\simeq 3839$ and $4300 \AA$ respectively) using VLT FORS2 spectra of 16 cluster members and find a sub-population of 5 stars enriched in nitrogen. We conclude that this is evidence for multiple populations in Kron 3, the fourth intermediate age cluster, after Lindsay 1, NGC 416 and NGC 339 (ages 6-8 Gyr), to display this phenomenon originally thought to be a unique characteristic of old globular clusters. At $\approx 6.5$ Gyr this is one of the youngest clusters with multiple populations, indicating that the mechanism responsible for their onset must operate until a redshift of at least 0.75 , much later than the peak of globular cluster formation at redshift $\sim 3$.
\end{abstract}

Key words: galaxies: Magellanic Clouds - galaxies: star clusters: individual: Kron 3

\section{INTRODUCTION}

Multiple populations (MPs) have been found in old, globular clusters (GCs, > 10 Gyr) in both the Milky Way (e.g.; Gratton et al. 2012) and the LMC (Mucciarelli et al. 2009; Mateluna et al. 2012) as well as the SMC (Dalessandro et al. 2016) and Fornax dwarf galaxy (Larsen et al. 2014). They are characterised spectroscopically by light element abundance variations and anticorrelations (e.g. O-Na anticorrelation; Carretta et al. 2009) and photometrically by spreads or splits in the main sequence and/or red giant branches in certain filters (e.g. Marino et al. 2008; Piotto et al. 2015).

The presence and mechanism behind the onset of MPs has significant consequences for globular cluster formation theories (e.g.; Decressin et al. 2007; D'Ercole et al. 2008; de Mink et al. 2009; Bastian et al. 2013). There are currently several proposed mechanisms for the formation of MPs, though all have difficulties recreating the required

* Based on observations made with ESO telescopes at the La Silla Paranal Observatory under Programme ID 096.B-0618(B).

† E-mail: kathie.hollyhead@astro.su.se) light element abundance variations that are observed across all GCs (Bastian et al. 2015; Renzini et al. 2015) and also run into other significant issues, the most prominent being the mass-budget problem (e.g. Larsen et al. 2012; Bastian \& Lardo 2015; Kruijssen 2015). Additionally, sufficient gas reservoirs that would be required for the formation of a second generation at the ages required by the current GC formation theories have also not been found in young massive clusters (Cabrera-Ziri et al. 2015; Longmore 2015). Having a working theory for the formation and evolution of globular clusters is important for overall galactic formation theories, particularly as populations of stars with similar properties have also been identified within the bulge of the Milky Way (Schiavon et al. 2017).

Mass has been found to be an important factor in whether MPs form in clusters (Carretta et al. 2010; Schiavon et al. 2013; Milone et al. 2017). The lowest mass Milky Way GC with MPs discovered recently is NGC 6535 at $10^{3.58} \mathrm{M}_{\odot}$ (Milone et al. 2017; Bragaglia et al. 2017), or potentially ESO452-SC11 at $6.8 \pm 3.4 \times 10^{3} \mathrm{M}_{\odot}$ (Simpson et al. 2017). However, massive clusters in the age range of 1-2 Gyr in the Magellanic Clouds of masses equal or greater than 
those of GCs have also been found to show a lack of evidence for MPs (e.g. NGC1806 and NGC419; Mucciarelli et al. 2014; Martocchia et al. 2017), indicating that age also plays an important role. Intriguingly, the phenomenon of extended main sequence turn-offs (eMSTOs) has been identified in clusters younger than 2 Gyr (e.g. Mackey et al. 2008), which were originally attributed to age spreads of $\sim 200-700$ Myr (e.g. Goudfrooij et al. 2014). However the magnitude of the spread was found to be proportional to the age of the cluster, and therefore unlikely to be real (Niederhofer et al. 2015; Milone et al. 2015). It is currently thought that stellar rotation can explain this observation (e.g. Bastian \& de Mink 2009; Niederhofer et al. 2015). Open clusters of comparable age (6-9 Gyr) and mass $\left(\sim 10^{4} \mathrm{M}_{\odot}\right)$ to GCs such as NGC 6791 or Berkeley 39 have also been found to lack MPs (Bragaglia et al. 2012, 2014; Cunha et al. 2015).

Until recently, there had existed a gap in the age ranges of clusters studied with the aim of looking for MPs, from $\approx 2-10$ Gyr. It was evident that observing massive clusters in this age range could help constrain the age at which MPs form and potentially the mechanism by which they are created. Lindsay 1, at $\sim 8$ Gyr old (Mighell et al. 1998; Glatt et al. 2008), was the first non-traditional ancient globular cluster to show evidence for MPs in the form of a statistically significant nitrogen spread compared to a negligible spread in carbon (Hollyhead et al. 2017). Six stars out of the 16 member stars were nitrogen enriched and belonged to a secondary sub-population within the cluster. This result was confirmed with HST photometry and a split in the RGB (Niederhofer et al. 2017b). Overlapping stars in the catalogues of the two studies indicated that stars with enhanced nitrogen lay on the secondary RGB when the cluster is imaged in a combination of special filters sensitive to $\mathrm{N}$ variations. In addition to Lindsay 1, NGC 416 and NGC 339 were then also found to host MPs from photometry (Niederhofer et al. 2017b).

In this paper we present the results for a further intermediate age, massive, metal-poor $\left(3.9-5.84 \times 10^{5} \mathrm{M}_{\odot}\right.$, $[\mathrm{Fe} / \mathrm{H}]=-1.08$ dex; Glatt et al. 2011) cluster in the SMC, Kron 3 (hereafter K3). At 6.5 Gyr old (Glatt et al. 2008) this is one of the youngest clusters with a detailed spectroscopic search for MPs, along with NGC 416 and NGC 339, both at $\sim 6$ Gyr (Glatt et al. 2008). In order to look for MPs we carried out a similar study to that of Lindsay 1 in Hollyhead et al. (2017) (hereafter H17) and obtained low resolution FORS2 spectra of 35 targets in the direction of the cluster. Using this data we calculate $\mathrm{CN}$ and $\mathrm{CH}$ band strengths (e.g. Kayser et al. 2008; Pancino et al. 2010; Lardo et al. 2012) and use stellar sythesis to find $[\mathrm{C} / \mathrm{Fe}]$ and $[\mathrm{N} / \mathrm{Fe}]$ to look for MPs.

In $\S 2$ we discuss our data taken using FORS2 on the VLT describing briefly how the spectra were reduced. $\S 3$ outlines our extensive and strict membership criteria for determining which of our stars belonged to K3 and we then outline our method for calculating $\mathrm{CN}$ and $\mathrm{CH}$ band strengths and abundances in $\S 4$. Finally, $\S 5$ shows our results for this cluster and in $\S 6$ we discuss our conclusions.

\section{OBSERVATIONS AND DATA REDUCTION}

Our data for K3 was taken during the same observing run as Lindsay 1, program ID 096.B-0618(B), P.I. N. Kacharov, as described in H17. Using multi-object spectroscopy with FORS2 on the VLT at the Paranal observatory in Chile, we obtained six science images centred on K3, along with bias frames and flat fields. It was necessary to use the GRIS $600 \mathrm{~B}+22$ grism to observe $\mathrm{CN}$ and $\mathrm{CH}$ bands at $3839 \AA$ and $4300 \AA$ respectively. Archival pre-imaging was available for photometry (ESO-programme: 082.B-0505(A) P.I. D. Geisler) in the $\mathrm{V}$ and $\mathrm{I}$ bands, for which we have errors of $\operatorname{sim} 0.05$. The resolution of the spectra is $R=\lambda / \Delta \lambda \simeq 800$, covering a nominal spectral range of $\sim 3300-6600 \AA$. The sampled spectral range varies from star to star and depends on their position on the instrument field of view.

Similarly as done for L1, we centered the master chip on the centre of the cluster, with the slave chip at the southern edge. 35 targets were also chosen for K3, with primary targets selected from the CMD sampling the lower RGB to avoid abundance contamination due to stellar evolution and the first dredge-up (Cohen et al. 2002; Kayser et al. 2008; Pancino et al. 2010). The centre of the cluster could not be sampled due to crowding.

Using IRAF, we reduced the spectra by subtracting bias frames, applying flat fields, and wavelength calibrating and extracting the 1D spectra. Cosmic rays were removed with the L.A.Cosmic IRAF routine (van Dokkum 2001). During extraction, the apertures showed little curvature, similar to L1 and so we allowed the apall task to account for this without applying an additional correction.

Fig. 1 shows example spectra of two member stars of K3. The bands adopted to measure $\mathrm{S} \lambda 3839$ and $\mathrm{CH} \lambda 4300$, and used for the spectral synthesis to measure $[\mathrm{C} / \mathrm{Fe}]$ and $[\mathrm{N} / \mathrm{Fe}]$ are highlighted on the spectra. We have chosen two stars with very similar effective temperatures and $\log (\mathrm{g})$ to show the differences in the $\mathrm{CN}$ band, which traces nitrogen.

\section{CLUSTER MEMBERSHIP}

Due to the low resolution of the spectra, it is more difficult to constrain cluster membership solely via radial velocity measurements. Therefore, we have employed a number of criteria to more accurately determine cluster membership as described in detail in H17. Fig. 2 and Fig. 3 show how we used our criteria to determine which stars are true cluster members.

Fig. 2 shows the radial velocities along with CaII $(\mathrm{H}+\mathrm{K})$ and Fe5270 band strength measurements (see $\S 4.1$ ) for all stars, plotted against their distances from the centre of the cluster in arcminutes. The first panel shows our radial velocities measured in fxcor in IRAF adopting a likely member star as a template with its radial velocity measured first with the rvidlines routine. The teal line indicates the previously observed velocity for K3 at $135.1 \mathrm{~km} / \mathrm{s}$ by Parisi et al. (2015). We decided on cluster members using a $1 \sigma$ $(\approx 30 \mathrm{~km} / \mathrm{s}$, which is also compatible with the precision of FORS2 with our setup) allowance from this value, indicated by the blue background. Filled symbols are members, while empty squares are outside of our range and considered nonmembers. Nine stars were removed using radial velocities. 

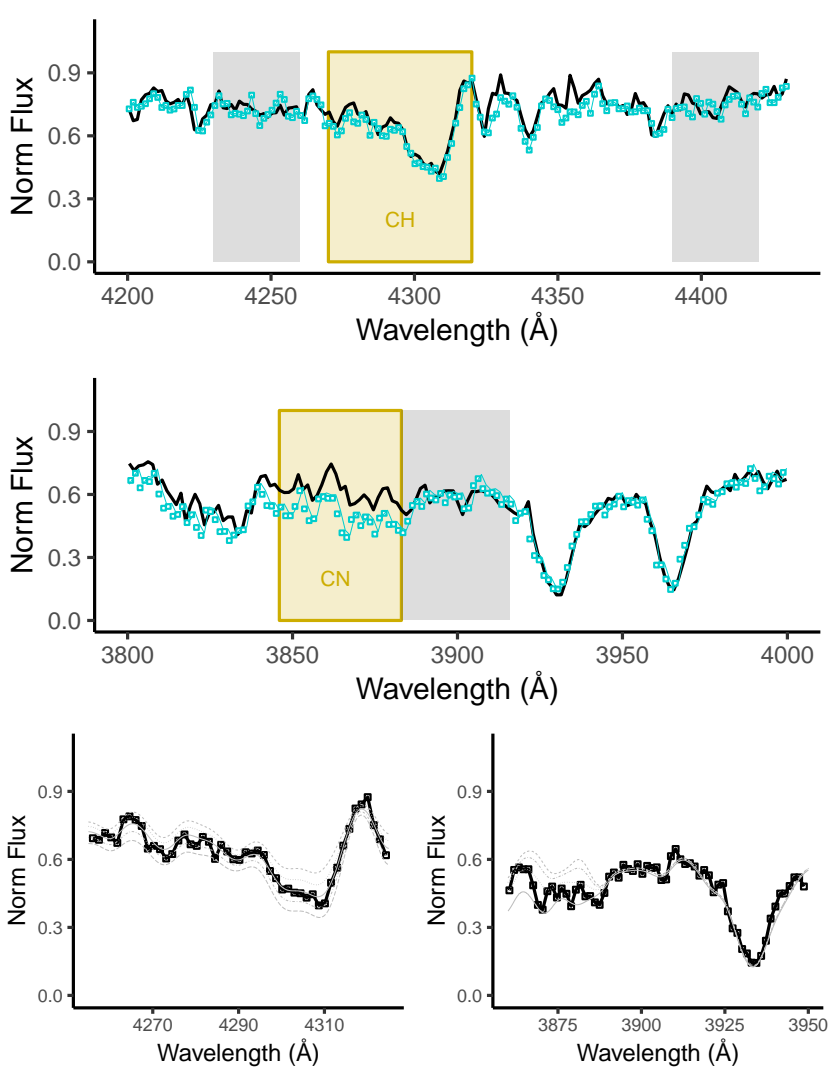

Figure 1. Example spectra of two member stars in K3 (stars 225 and 3910). The stars have similar Teff and $\log (\mathrm{g})$ to illustrate the difference seen in the $\mathrm{CN}$ band at $3839 \AA$ and the similarity in the $\mathrm{CH}$ band at $4300 \mathrm{~A}$. The yellow regions represent the spectral regions from which we measured the $\mathrm{CN}$ and $\mathrm{CH}$ indices. The grey areas are the respective continuum windows. The bottom left panel shows observed (black) and synthetic (grey) spectra around $\mathrm{CH}$ band for the star 3910 . The grey lines are the syntheses computed with $\mathrm{C}$ abundance altered by $-0.8,-0.6,-0.4,-0.2,+0.0$ dex (from bottom to top). The bottom right panel shows the same as the left hand panel but for the CN feature. The synthetic spectra show the syntheses computed with $\mathrm{N}$ abundance altered by $-0.8,-0.4,+0.0,+0.6$ dex (from bottom to top).

The second panel shows CaII $(\mathrm{H}+\mathrm{K})$ index measurements for all stars. In this case we used a $2 \sigma(\approx 9 \mathrm{mag})$ cut off from the median for member stars. Two stars were identified as non-members from this index. Finally, the lowest panel shows the Fe5270 band strengths. Again a $2 \sigma(\approx 0.05$ mag) cut was used and two stars were identified. Many of the criteria had overlapping stars identified as non-members. The spread in these measurements is larger than we found for L1, where we were able to use a cut-off of $1 \sigma$ from the median of both Fe5270 and CaII $(\mathrm{H}+\mathrm{K})$. With K3, however, using $1 \sigma$ would remove potential cluster members and many more than those outside of the acceptable radial velocity range. Therefore we use $2 \sigma$ as our range for members.

Fig. 3 shows the CMD for all stars in the photometry of K3, with stars which survived our previous selection showed as filled symbols. All stars lie on the RGB, however we removed the bright star 791 at $V \sim 19.7$ as it could be more strongly affected by evolutionary mixing due to its luminos-
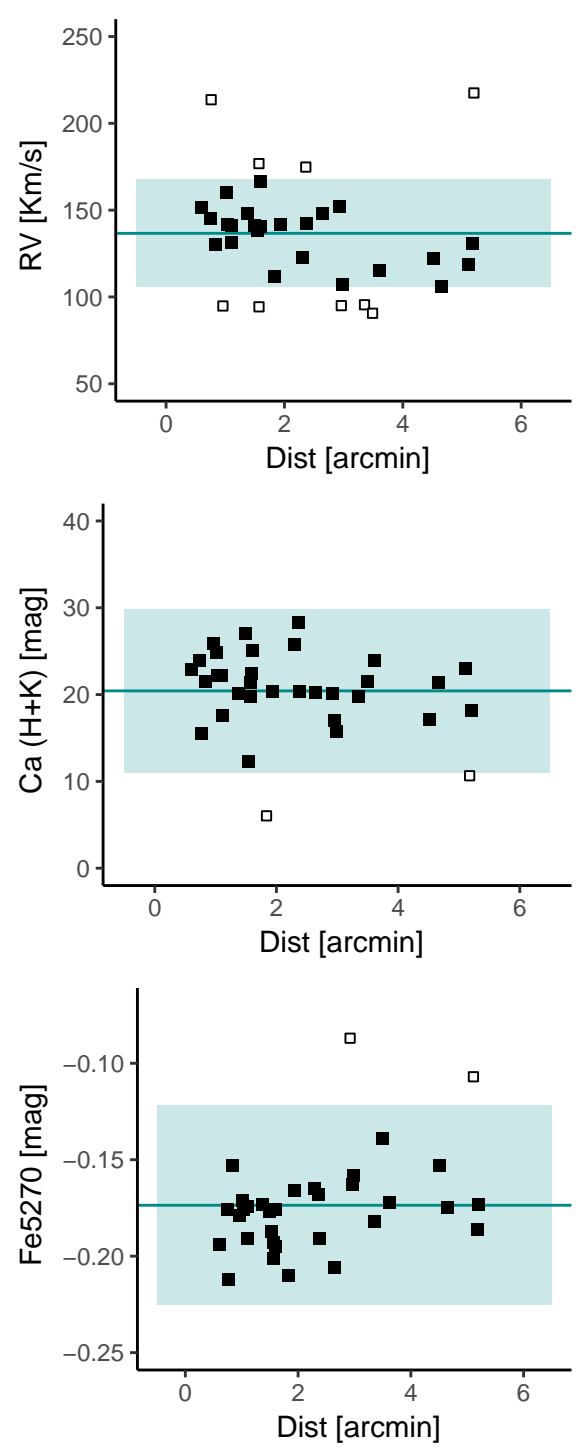

Figure 2. The radial velocity, CaII $(H+K)$ and Fe5270 measurements for all stars against their distances from the centre of the cluster in arcminutes. Filled symbols are considered members and empty ones non-members. 9 stars were removed due to outlying radial velocities, two to CaII $(\mathrm{H}+\mathrm{K})$ and two from Fe5270, though several of these overlap.

ity (Gratton et al. 2000; Angelou et al. 2015; Dotter et al. 2017).

Finally, spectra with $\mathrm{S} / \mathrm{N} \leq 10$ (per pixel) in the $\mathrm{CN}$ band region were rejected. Spectra with significant defects (spikes, holes) in the measurement windows were also rejected. These cuts left 16 member stars for K3 across both chips (see Table 1).

\section{INDEX MEASUREMENT AND SPECTRAL SYNTHESIS}

\subsection{CN and $\mathrm{CH}$ band strengths}

As per $\mathrm{H} 17$ we calculated the $U V \mathrm{CN}$ and $\mathrm{G} \mathrm{CH}$ band strengths $(\mathrm{S}(\lambda 3839)$ and $\mathrm{CH}(\lambda 4300)$ respectively) using the definitions from Norris et al. (1981); Worthey (1994) and 


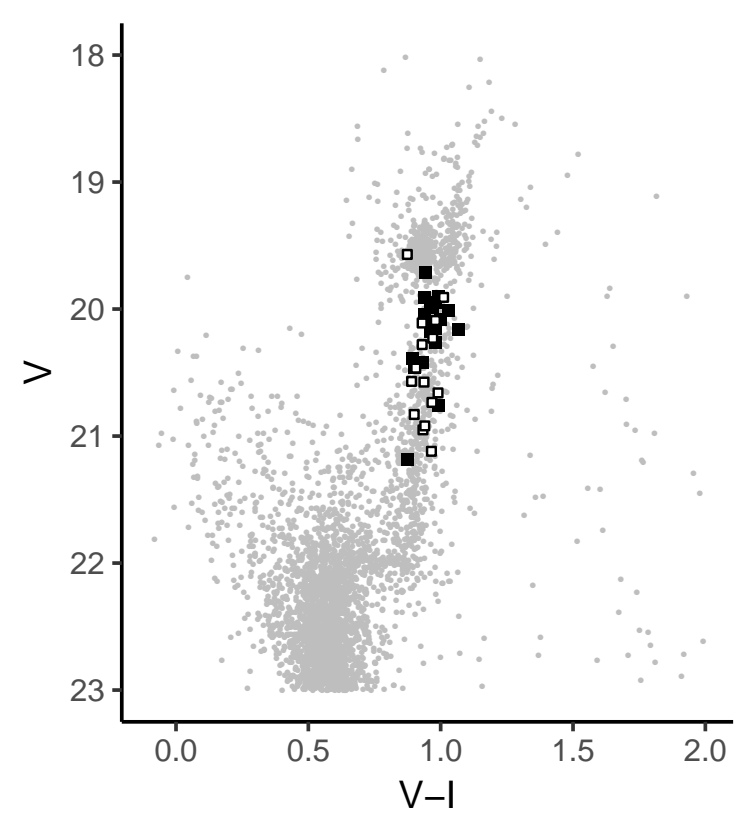

Figure 3. One of our criteria used to determine cluster membership. The plot shows the CMD for all stars in our analysis with stars which survived our selection based on radial velocity, CaII $(\mathrm{H}+\mathrm{K})$ and Fe5270 measurements as filled symbols. Empty symbols are rejected stars. All stars appear to lie on the RGB, however the star at $V \sim 19.7$ was removed due to its high luminosity and the possibility of it being affected by mixing.

Lardo et al. (2013) with error measurements estimated as per Vollmann \& Eversberg (2006). The average S/N for the $\mathrm{CN}$ and $\mathrm{CH}$ bands is $\sim 25$ and $\sim 40$, respectively. As discussed in e.g. Lardo et al. (2012), the CH band at $4300 \AA$ is not affected by the change in spectral slope from atmosphere or instrumental effects thanks to two continuum bandpasses. On the other hand, one has to rely only on a single continuum bandpass in the red part of the spectral feature for the $3883 \AA$ CN band (see Fig. 1). Following Cohen et al. (2002, 2005), we normalised the stellar continuum in the spectrum of each star and then measured the absorption within both the $\mathrm{CH}$ and $\mathrm{CN}$ bandpasses. The polynomial fitting used a $6 \sigma$ high and $3 \sigma$ low clipping, running over a five pixel average. By fitting the continuum, we were able to directly compare the indices measured in this section and the abundances derived from spectral synthesis in Sect. 4.2.

Fe5270 and CaII $(\mathrm{H}+\mathrm{K})$ used previously to constrain membership in $\S 3$ were calculated in a similar fashion.

Index measurements for all member stars in K3 are listed in Table 1.

\section{$4.2[\mathrm{C} / \mathrm{Fe}]$ and $[\mathrm{N} / \mathrm{Fe}]$}

In addition to using the $\mathrm{CN}$ and $\mathrm{CH}$ band strengths to investigate multiple populations in $\mathrm{K} 3$, we also used spectral synthesis to quantify $[\mathrm{C} / \mathrm{Fe}]$ and $[\mathrm{N} / \mathrm{Fe}]$ for the stars to look for evidence of an enriched population. We first evaluated effective temperatures $\left(T_{\text {eff }}\right)$ using the $(V-I)$ colour in

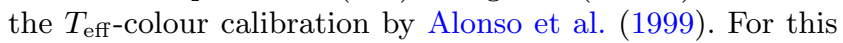
calibration we adopted $[\mathrm{Fe} / \mathrm{H}]=-1.08$ from Da Costa \& Hatzidimitriou (1998) and $E(V-I)=0.008$ from Glatt et al.
(2008). We then calculated the surface gravity for each star using the previously derived $T_{\text {eff }}$ with a distance modulus of 18.8 (Glatt et al. 2008), the assumption of a mass of $0.95 \mathrm{M}_{\odot}$ from BaSTI isochrones and the bolometric corrections from Alonso et al. (1999). Finally, a microturbulent velocity of $v_{t}=2.0 \mathrm{kms}^{-1}$ was assigned to all stars.

Stellar abundances for $[\mathrm{C} / \mathrm{Fe}]$ and $[\mathrm{N} / \mathrm{Fe}]$ were calculated in the same way as in H17. We took line lists (both atomic and molecular) from the most recent Kurucz compilation from the website of F. Castelli ${ }^{1}$. The grid of models used as starting points to determine model atmospheres was also taken from this website. The atmospheres were then calculated using the ATLAS9 code (Castelli \& Kurucz 2004) with the previously quoted values for $[\mathrm{Fe} / \mathrm{H}], T_{\text {eff }}$, $\mathrm{v}_{\text {mic }}, \log (\mathrm{g})$ and a solar-scaled composition.

Model spectra with a range of abundances were generated with the SYNTHE code by Kurucz and subsequently fitted to our observations finding the best fit with a $\chi^{2}$ minimisation routine and thereby deriving $[\mathrm{C} / \mathrm{Fe}]$ and $[\mathrm{N} / \mathrm{Fe}]$ with solar abundances taken from Asplund et al. (2009). A fit was determined by minimising the observed-computed spectrum difference in a $100 \AA$ window centred on $4300 \AA$ for the $\mathrm{CH}$ G-band and $50 \AA$ window for the UV CN feature at $3883 \AA$. Running SYNTHE on quite a broad spectral range of about $200 \AA$ to produce synthetic spectra allowed us to set a reasonable continuum level also by visual inspection and thus compute robust abundances. It was necessary to use an iterative method as the $\mathrm{C}$ abundance must be known prior to determining that of $\mathrm{N}$, as both contribute to the $\mathrm{CN}$ band.

We adopted a constant oxygen abundance $([\mathrm{O} / \mathrm{Fe}]=$ $+0.2 \mathrm{dex})$ throughout all computations. The derived $\mathrm{C}$ abundance is dependent on the $\mathrm{O}$ abundance and therefore so is the $\mathrm{N}$ abundance. To test the sensitivity of the $\mathrm{C}$ abundance to the adopted $\mathrm{O}$ abundance we varied the oxygen abundances and repeated the spectrum synthesis to determine the exact dependence for the coolest and warmest stars in our sample (with $T_{\text {eff }} \sim 4600$ and 5000, respectively. In these computations, we adopted $[\mathrm{O} / \mathrm{Fe}]=0.0$ and +0.4 dex. We found that significantly large variations in the oxygen abundance slightly affect $(\delta \mathrm{A}(\mathrm{C}) / \delta[\mathrm{O} / \mathrm{Fe}] \simeq 0.05 \mathrm{dex})$ the derived $\mathrm{C}$ abundance in cooler stars $\left(T_{\text {eff }} \sim 4600-4800\right)$, while they are negligible (of the order of 0.02 dex or less) for hotter stars. The total error in the $\mathrm{A}(\mathrm{C})$ and $\mathrm{A}(\mathrm{N})$ abundance was computed by taking into account the two main sources of uncertainty: (1) the error in the adopted atmospheric parameters and (2) the error in the fitting procedure (continuum placement and random noise). Errors in the adopted $T_{\text {eff }}$ translate to an uncertainty of $\delta \mathrm{A}(\mathrm{C}) / \delta T_{\text {eff }}$ $\simeq 0.05-0.10$ dex and $\delta \mathrm{A}(\mathrm{N}) / \delta T_{\text {eff }} \simeq 0.06-0.12$ in the $\mathrm{C}$ and $\mathrm{N}$ abundances (similar for the hottest and coolest stars in our sample). The errors due to uncertainties on gravity are negligible (on the order of 0.06 dex or less) and those due to continuum placement are of the order of $\sim 0.15$ dex. To evaluate intrinsic errors on $\mathrm{C}$ and $\mathrm{N}$ measurement abundances the fitting procedure is repeated for a sample of 500 synthetic spectra where Poissonian noise has been introduced to reproduce the observed noise conditions. The errors derived from the fitting procedure were then added in quadrature to

\footnotetext{
1 http://wwwuser.oats.inaf.it/castelli/linelists.html
} 

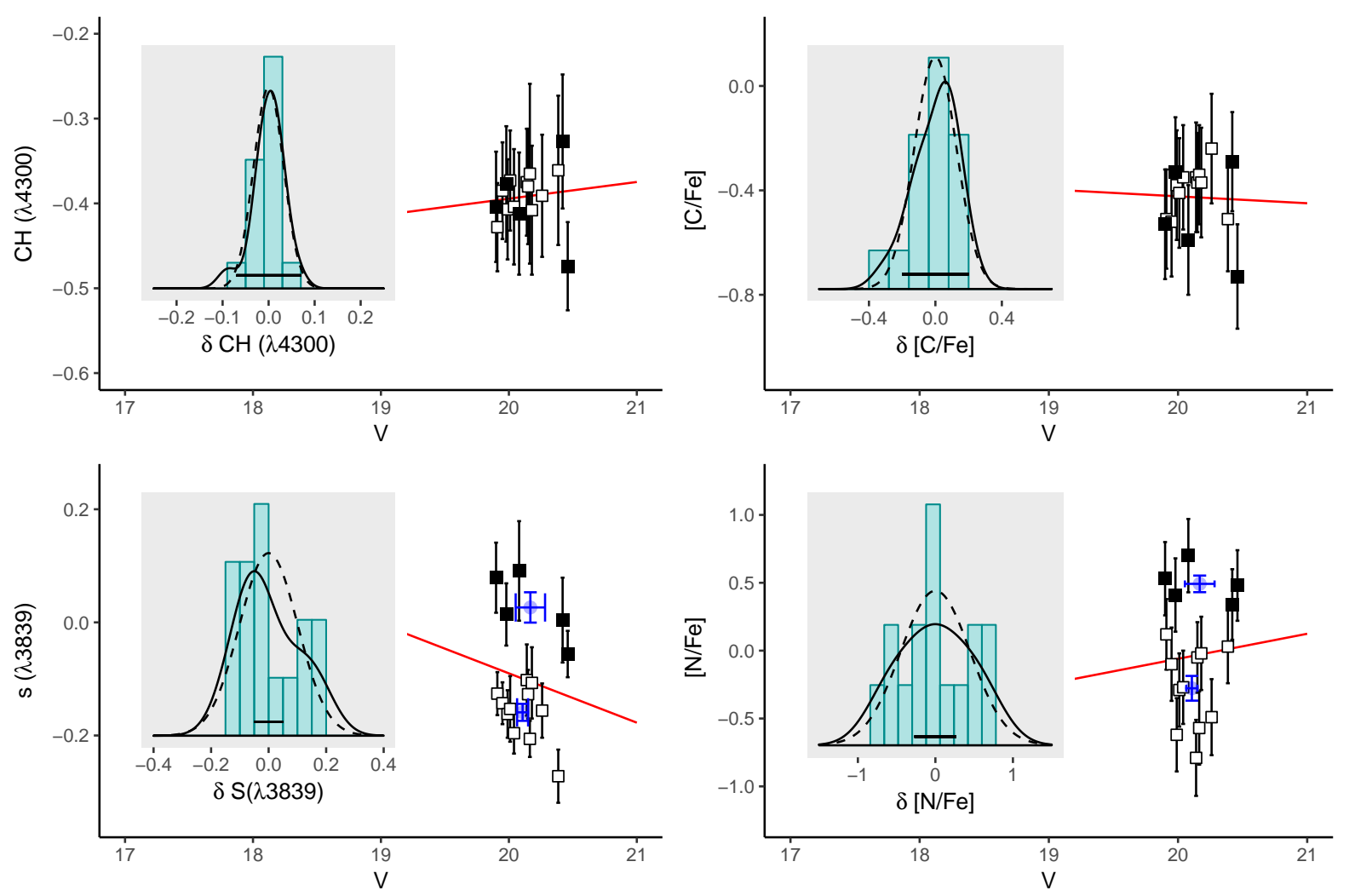

Figure 4. From top to bottom, left to right: The run of $\mathrm{CN}, \mathrm{CH},[\mathrm{C} / \mathrm{Fe}]$, and $[\mathrm{N} / \mathrm{Fe}]$ is plotted against the apparent $\mathrm{V}$ band magnitude for all member stars. The red lines indicate the linear fit of those quantities vs. the visual magnitude. CN-strong and CN-weak stars are plotted as filled and empty symbols, respectively. The mean $\mathrm{CN}$ index and $[\mathrm{N} / \mathrm{Fe}]$ abundance of the $\mathrm{CN}$-strong and $\mathrm{CN}$-weak groups and the associated standard errors are plotted in blue. The insets show the histograms and the associated kernel distributions (solid black line) of the residuals. The dashed line represents the Gaussian distribution that best fit the data. The mean errors associated with the measurements are also plotted at the base of each histogram.

the errors introduced by atmospheric parameters, resulting in an overall error of $\sim \pm 0.20$ dex for the $\mathrm{C}$ abundances and $\sim \pm 0.27$ dex for the $\mathrm{N}$ values.

A combination of these errors was used as the final error estimation for each abundance measurement, as given in Table. 1.

\section{RESULTS}

In Fig. 4 we plot the $\mathrm{CN}$ and $\mathrm{CH}$ indexes along with the associated $[\mathrm{C} / \mathrm{Fe}]$ and $[\mathrm{N} / \mathrm{Fe}]$ abundance ratios for each of our member stars vs. their apparent $\mathrm{V}$ band magnitudes. The insets show the histograms of the residuals of the linear fit of those quantities vs. the visual magnitude. In the case of both the $\mathrm{CH}$ index and $[\mathrm{C} / \mathrm{Fe}]$ abundance ratio, the derived spread is very small and within the uncertainties. Thus, no significant carbon variation among target stars can be deduced from the data.

Conversely, a visual inspection of the bottom left hand panel of Fig 5 reveals a statistically significant bimodality in the $\mathrm{CN}$ index over the whole sampled magnitude range. $\mathrm{CN}$-strong and $\mathrm{CN}$-weak stars are observed at the same magnitude, indicating that the observed star-to-star variation in $\mathrm{CN}$ must be intrinsic. Indeed, the observed bimodality in $\mathrm{CN}$ (as well as the elevated nitrogen abundances) can- not be explained by evolutionary mixing as they are fainter than the luminosity function bump $\left(V_{B U M P}=19.38 \pm 0.04\right.$; Alves \& Sarajedini 1999). Therefore, any mixing with evolution should have had little impact on our abundances for both carbon and nitrogen (e.g. Gratton et al. 2000). An increase in $\mathrm{CN}$ band strength with increasing luminosity is possible, but such an observed trend relies only on a few targets at the faintest magnitude end. Using this plot we are able to clearly distinguish between the enriched (i.e. CNstrong stars, shown as filled symbols) and CN-weak (i.e. nonenriched, shown as empty symbols) component. The mean $\mathrm{CN}$ index and $[\mathrm{N} / \mathrm{Fe}]$ abundance were then computed within each subpopulation. The difference in $\mathrm{S}(\lambda 3839)$ between $\mathrm{CN}$ strong and CN-weak stars of comparable magnitude is $\sim 0.2$ mag.

CN-strong stars tend also to have on average higher $[\mathrm{N} / \mathrm{Fe}]$ abundance than $\mathrm{CN}$-weak stars, with the mean $[\mathrm{N} / \mathrm{Fe}]$ abundance of the two groups $\sim+0.5$ and -0.3 dex respectively. In this case, because we observe a large spread rather than an obvious bimodality, computing the mean abundance of subpopulations split according to their CN index will tend to exaggerate the differences between the two populations. However, to assess the statistical significance of the internal nitrogen variation, we consider also the stars at the extremes of the distribution. Stars with the highest and lowest $[\mathrm{N} / \mathrm{Fe}]$ abundances differ at more than $3 \sigma$ level. There 
is therefore additional evidence for internal variation in nitrogen in K3 stars also from nitrogen abundances despite the larger uncertainties associated to $[\mathrm{N} / \mathrm{Fe}]$ measurements. The enhancement of the UV CN band which is observed in the $\mathrm{CN}$-strong population with respect to $\mathrm{CN}$-weak one cannot have been misinterpreted because of errors in the modelatmosphere abundance analysis, as shown in Fig. 1, where we plot the spectra of two stars with similar atmospheric parameters yet different strength of their $\mathrm{CN}$ absorption.

Both band strengths and abundances show the same trend: a negligible spread in carbon compared to a significant spread in nitrogen, which is greater than the errors. Again, the filled symbols indicate those that we would consider enriched in nitrogen. We conclude that the presence of such a CN-strong sub-population is a strong indication that multiple populations are present in Kron 3, as shown for Lindsay 1 in H17, as well as globular clusters in other galaxies (Kayser et al. 2008). The spread in nitrogen of up to 1 dex in Kron 3 is comparable to that of Lindsay 1, for which however, we were not able to detect a clear bimodality in the $\mathrm{CN}$ absorption bands.

Out of a sample of 16 stars, 5 have strong CN bands. This could suggest that the majority of stars belong to the non-enriched population, contrary to what is observed in Galactic GCs with present mass similar to that of K3. However, the spectroscopic sample presented here may be not representative of the entirety of the cluster, as the statistics are poor (16 stars) and the radial coverage is severely biased towards the outskirts of the cluster due to crowding in the central regions. From larger photometric samples, Niederhofer et al. (2017b) find that the fraction of enriched stars ranges from $24 \%$ to $45 \%$ in three SMC clusters with masses and ages similar to those of K3 (ages 6-8 Gyr, M $10^{5} \mathrm{M}_{\odot}$ ). Dalessandro et al. (2016) and Niederhofer et al. (2017a) find that the non-enriched population account for more than $65 \%$ of the total cluster mass also in NGC 121, the oldest cluster in the SMC at 11 Gyr, although due to differing methods, the fraction is not directly comparable to that of the HST UV Legacy Sample (Milone et al. 2017). Our result agrees well with the number ratios given by Niederhofer et al. (2017a,b) and Dalessandro et al. (2016), but more studies are needed to confirm whether the observed paucity of enriched stars in the SMC clusters with respect to the Milky Way clusters can be considered a general characteristic of the SMC cluster population.

In Fig. 5 we plot the run of the $\mathrm{CN}$ band vs. the $\mathrm{CH}$ absorption (top panel) and the $[\mathrm{N} / \mathrm{Fe}]$ abundance ratios vs. $[\mathrm{C} / \mathrm{Fe}]$ (bottom panel). The mean values along with the associated standard errors are also indicated. From this plot we note that $\mathrm{CN}$-strong stars are not necessarily $\mathrm{CH}$-weak (i.e. $\mathrm{C}$ and $\mathrm{N}$ are not anti-correlated, see also the top panel of Fig. 4. This could possibly indicate that the nitrogen variations are not necessarily associated with carbon variations.

Such a trend was already observed in L1 and also in Milky Way GCs by Mészáros et al. (2015) from APOGEE data (Majewski et al. 2017). The former find that a statistically significant anti-correlation between $[\mathrm{C} / \mathrm{Fe}]$ and $[\mathrm{N} / \mathrm{Fe}]$ cannot be seen in any of the studied clusters, while a clear bimodality in $\mathrm{CN}$ can be observed for the most metal-rich $([\mathrm{Fe} / \mathrm{H}] \leq-1.5 \mathrm{dex})$ clusters of the sample. This seems to be the case of both $\mathrm{K} 3$ and $\mathrm{L} 1$. Our $[\mathrm{C} / \mathrm{Fe}]$ abundance ratio determinations have large associated uncertainty of $\sim 0.20$
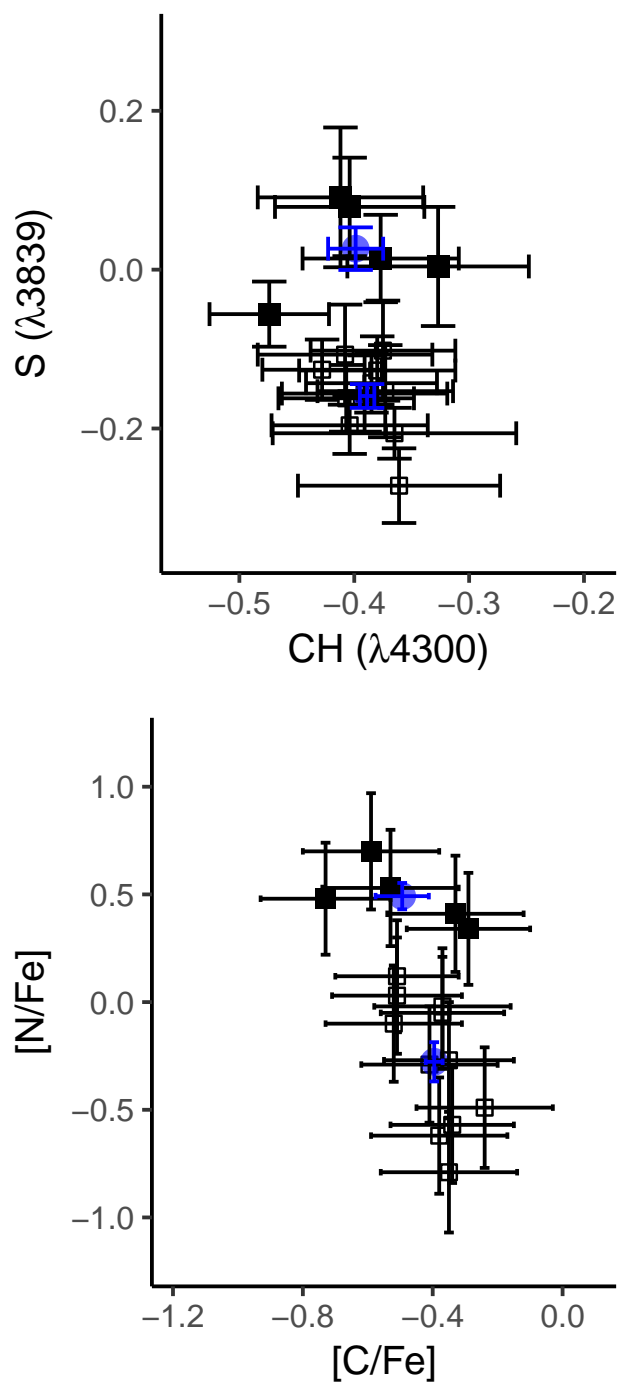

Figure 5. The top plot shows $\mathrm{CH}(\lambda 4300)$ against $\mathrm{S}(\lambda 3839)$ band strengths tracing nitrogen and carbon respectively for all member stars, while the bottom plot shows the same stars' $[\mathrm{C} / \mathrm{Fe}]$ against $[\mathrm{N} / \mathrm{Fe}]$. In both cases, there is clearly a larger spread in nitrogen than carbon. Mean abundances are also shown, along with their associated standard errors. Symbols are the same as Fig. 4.

dex. If the anti-correlations exist, they must span ranges smaller than our uncertainties in $[\mathrm{C} / \mathrm{Fe}]$. In general (within ancient GCs), nitrogen spans very large ranges (up to $\Delta$ $[\mathrm{N} / \mathrm{Fe}] \sim 2 \mathrm{dex}$, while $[\mathrm{C} / \mathrm{Fe}]$ variations are usually smaller than $\Delta[\mathrm{C} / \mathrm{Fe}] \sim 0.5$ dex (see for example figures 10 and 11 in Cohen et al. 2005). Similar to Mészáros et al. (2015), we believe that the lack of any clear evidence of carbon variations is due to the spread being smaller than the precision of the instrument observing very faint stars.

\section{DISCUSSION AND CONCLUSIONS}

FORS2 spectroscopy of 35 RGB stars in the intermediate age massive cluster Kron 3 in the SMC have yielded 16 member stars after a conservative membership selection process. We were able to derive $\mathrm{CN}$ and $\mathrm{CH}$ index measurements 
and $\mathrm{C}$ and $\mathrm{N}$ abundances for 16 stars. Five stars out of 16 show nitrogen enrichment. Figs. 4 and 5 indicate a significant spread in both $\mathrm{S} \lambda 3839$ and $[\mathrm{N} / \mathrm{Fe}]$, larger than the errors, compared to a relatively constant $\mathrm{CH} \lambda 4300$ and $[\mathrm{C} / \mathrm{Fe}]$. As with our previous study of a similar intermediate age massive cluster in the SMC, Lindsay 1 (H17), we suggest that this spread is indicative of the presence of multiple populations. At 6.5 Gyr, Kron 3 is one of the youngest clusters to show these variations, which means that the still-unknown mechanism responsible for the onset of MPs operates until a redshift of at least 0.75 , much later than the peak of globular cluster formation at redshift of $\approx 3$. This means the mechanism could still be working to create multiple populations in young massive clusters, so they can be considered analogues to GCs and used to constrain GC formation theories.

Multiple populations are usually identified using high resolution spectra yielding abundances of elements, most commonly $[\mathrm{O} / \mathrm{Fe}]$ and $[\mathrm{Na} / \mathrm{Fe}]$ (Carretta et al. 2009). Our method using low resolution spectra and band strengths is also routinely used to study MPs in ancient GCs (e.g. Kayser et al. 2008; Martell et al. 2008; Pancino et al. 2010). This method has some advantages over high resolution spectroscopy, with a simpler and quicker method for obtaining results and the ability to study the presence of MPs in clusters at greater distances that are too faint to be studied at high resolution with a reasonable observing time. To ensure that our results are reliable, we have used strict membership criteria as detailed in $\S 3$ and ensured our abundances and band strengths are not affected by effects such as evolutionary mixing.

This method has already been validated in the past for the old, low mass stars: the MW GCs that show both photometric evidence of multiple populations and have spectroscopically confirmed presence of MPs through $\mathrm{Na}-\mathrm{O}$ and similar light element anti-correlations have been shown to present variations in $\mathrm{CN}$ index and/or $[\mathrm{N} / \mathrm{Fe}]$ spread (e.g. Pancino et al. 2010). Furthermore, this method was used to detect MPs in the intermediate-age cluster L1, which has younger and thus more massive RGB stars than typical ancient GCs. The spread in nitrogen reported by H17 was confirmed to correspond to different populations via HST photometry (Niederhofer et al. 2017b). This strengthens the reliability of this method and our conclusion that Kron 3 hosts multiple populations.

\section{ACKNOWLEDGEMENTS}

C.L. thanks the Swiss National Science Foundation for supporting this research through the Ambizione grant number PZ00P2 168065.

E.K.G. gratefully acknowledges support from the German Research Foundation (DFG) via Sonderforschungsbereich SFB 881 ("The Milky Way System", subproject A8).

\section{REFERENCES}

Alonso A., Arribas S., Martínez-Roger C., 1999, A\&AS, 140, 261 Alves D. R., Sarajedini A., 1999, ApJ, 511, 225

Angelou G. C., D'Orazi V., Constantino T. N., Church R. P., Stancliffe R. J., Lattanzio J. C., 2015, MNRAS, 450, 2423
Asplund M., Grevesse N., Sauval A. J., Scott P., 2009, ARA\&A, 47,481

Bastian N., Lardo C., 2015, MNRAS, 453, 357

Bastian N., de Mink S. E., 2009, MNRAS, 398, L11

Bastian N., Lamers H. J. G. L. M., de Mink S. E., Longmore S. N., Goodwin S. P., Gieles M., 2013, MNRAS, 436, 2398

Bastian N., Cabrera-Ziri I., Salaris M., 2015, MNRAS, 449, 3333

Bragaglia A., Gratton R. G., Carretta E., D’Orazi V., Sneden C., Lucatello S., 2012, A\&A, 548, A122

Bragaglia A., Sneden C., Carretta E., Gratton R. G., Lucatello S., Bernath P. F., Brooke J. S. A., Ram R. S., 2014, ApJ, 796, 68

Bragaglia A., Carretta E., D'Orazi V., Sollima A., Donati P., Gratton R. G., Lucatello S., 2017, preprint, (arXiv:1708.07705)

Cabrera-Ziri I., et al., 2015, MNRAS, 448, 2224

Carretta E., et al., 2009, A\&A, 505, 117

Carretta E., Bragaglia A., Gratton R. G., Recio-Blanco A., Lucatello S., D’Orazi V., Cassisi S., 2010, A\&A, 516, A55

Castelli F., Kurucz R. L., 2004, ArXiv Astrophysics e-prints,

Cohen J. G., Briley M. M., Stetson P. B., 2002, AJ, 123, 2525

Cohen J. G., Briley M. M., Stetson P. B., 2005, AJ, 130, 1177

Cunha K., et al., 2015, ApJ, 798, L41

D'Ercole A., Vesperini E., D'Antona F., McMillan S. L. W., Recchi S., 2008, MNRAS, 391, 825

Da Costa G. S., Hatzidimitriou D., 1998, AJ, 115, 1934

Dalessandro E., Lapenna E., Mucciarelli A., Origlia L., Ferraro F. R., Lanzoni B., 2016, ApJ, 829, 77

Decressin T., Meynet G., Charbonnel C., Prantzos N., Ekström S., 2007, A\&A, 464, 1029

Dotter A., Conroy C., Cargile P., Asplund M., 2017, ApJ, 840, 99

Glatt K., et al., 2008, AJ, 136, 1703

Glatt K., et al., 2011, AJ, 142, 36

Goudfrooij P., et al., 2014, ApJ, 797, 35

Gratton R. G., Sneden C., Carretta E., Bragaglia A., 2000, A\&A, 354,169

Gratton R. G., Carretta E., Bragaglia A., 2012, A\&ARv, 20, 50

Hollyhead K., et al., 2017, MNRAS, 465, L39

Kayser A., Hilker M., Grebel E. K., Willemsen P. G., 2008, A\&A, 486, 437

Kruijssen J. M. D., 2015, MNRAS, 454, 1658

Lardo C., et al., 2012, A\&A, 541, A141

Lardo C., et al., 2013, MNRAS, 433, 1941

Larsen S. S., Strader J., Brodie J. P., 2012, A\&A, 544, L14

Larsen S. S., Brodie J. P., Grundahl F., Strader J., 2014, ApJ, 797,15

Longmore S. N., 2015, MNRAS, 448, L62

Mackey A. D., Broby Nielsen P., Ferguson A. M. N., Richardson J. C., 2008, ApJ, 681, L17

Majewski S. R., et al., 2017, AJ, 154, 94

Marino A. F., Villanova S., Piotto G., Milone A. P., Momany Y., Bedin L. R., Medling A. M., 2008, A\&A, 490, 625

Martell S. L., Smith G. H., Briley M. M., 2008, PASP, 120, 7

Martocchia S., et al., 2017, MNRAS, 468, 3150

Mateluna R., Geisler D., Villanova S., Carraro G., Grocholski A., Sarajedini A., Cole A., Smith V., 2012, A\&A, 548, A82

Mészáros S., et al., 2015, AJ, 149, 153

Mighell K. J., Sarajedini A., French R. S., 1998, AJ, 116, 2395

Milone A. P., et al., 2015, MNRAS, 450, 3750

Milone A. P., et al., 2017, MNRAS, 464, 3636

Mucciarelli A., Origlia L., Ferraro F. R., Pancino E., 2009, ApJ, 695, L134

Mucciarelli A., Dalessandro E., Ferraro F. R., Origlia L., Lanzoni B., 2014, ApJ, 793, L6

Niederhofer F., Georgy C., Bastian N., Ekström S., 2015, MNRAS, 453, 2070

Niederhofer F., et al., 2017a, MNRAS, 464, 94

Niederhofer F., et al., 2017b, MNRAS, 465, 4159 
Norris J., Cottrell P. L., Freeman K. C., Da Costa G. S., 1981, ApJ, 244, 205

Pancino E., Rejkuba M., Zoccali M., Carrera R., 2010, A\&A, 524, A44

Parisi M. C., Geisler D., Clariá J. J., Villanova S., Marcionni N., Sarajedini A., Grocholski A. J., 2015, AJ, 149, 154

Piotto G., et al., 2015, AJ, 149, 91

Renzini A., et al., 2015, MNRAS, 454, 4197

Schiavon R. P., Caldwell N., Conroy C., Graves G. J., Strader J., MacArthur L. A., Courteau S., Harding P., 2013, ApJ, 776, L7

Schiavon R. P., et al., 2017, MNRAS, 465, 501

Simpson J. D., De Silva G., Martell S. L., Navin C. A., Zucker D. B., 2017, preprint, (arXiv:1708.06875)

Vollmann K., Eversberg T., 2006, Astronomische Nachrichten, 327,862

Worthey G., 1994, ApJS, 95, 107

de Mink S. E., Pols O. R., Langer N., Izzard R. G., 2009, A\&A, 507, L1

van Dokkum P. G., 2001, PASP, 113, 1420

This paper has been typeset from a $\mathrm{T}_{\mathrm{E}} \mathrm{X} / \mathrm{LAT}_{\mathrm{E}} \mathrm{X}$ file prepared by the author. 
Stellar properties

\begin{tabular}{|c|c|c|c|c|c|c|c|c|c|c|c|c|c|c|c|c|c|c|c|c|c|}
\hline Star & $\begin{array}{c}\text { R.A. } \\
\text { (Degrees) }\end{array}$ & $\begin{array}{c}\text { Dec } \\
\text { (Degrees) }\end{array}$ & $\begin{array}{c}\mathrm{V} \\
(\mathrm{mag})\end{array}$ & $\begin{array}{l}\mathrm{T}_{\text {eff }} \\
(\mathrm{K})\end{array}$ & $\begin{array}{l}\mathrm{eT}_{\text {eff }} \\
(\mathrm{K})\end{array}$ & $\begin{array}{r}\log (g) \\
(\operatorname{dex})\end{array}$ & $\begin{array}{c}\operatorname{elog}(g) \\
(\operatorname{dex})\end{array}$ & $\begin{array}{c}{[\mathrm{C} / \mathrm{Fe}]} \\
(\mathrm{dex})\end{array}$ & $\begin{array}{c}\mathrm{e}[\mathrm{C} / \mathrm{Fe}] \\
(\mathrm{dex})\end{array}$ & $\begin{array}{r}{[\mathrm{N} / \mathrm{Fe}]} \\
(\mathrm{dex})\end{array}$ & $\begin{array}{c}\mathrm{e}[\mathrm{N} / \mathrm{Fe}] \\
(\mathrm{dex})\end{array}$ & $\begin{array}{r}\mathrm{CN} \\
(\mathrm{mag})\end{array}$ & $\begin{array}{l}\mathrm{eCN} \\
(\mathrm{mag})\end{array}$ & $\begin{array}{c}\mathrm{CH} \\
(\mathrm{mag})\end{array}$ & $\begin{array}{c}\mathrm{eCH} \\
(\mathrm{mag})\end{array}$ & $\begin{array}{c}\mathrm{Ca}(\mathrm{HK}) \\
\text { (mag) }\end{array}$ & $\begin{array}{r}\mathrm{eCa} \\
(\mathrm{mag})\end{array}$ & $\begin{array}{c}\mathrm{Fe} \\
(\mathrm{mag})\end{array}$ & $\begin{array}{c}\mathrm{eFe} \\
(\mathrm{mag})\end{array}$ & $\begin{array}{r}\mathrm{RV} \\
(\mathrm{km} / \mathrm{s})\end{array}$ & Notes \\
\hline 225 & 6.135502 & -72.82457 & 19.99 & 4837 & 90 & 2.4 & 0.2 & -0.38 & 0.21 & -0.62 & 0.27 & -0.162 & 0.042 & -0.407 & 0.059 & 25.773 & 6.265 & -0.165 & 0.03 & 123.0 & $1 \mathrm{P}$ \\
\hline 419 & 6.298478 & -72.85623 & 20.16 & 4607 & 78 & 2.4 & 0.2 & -0.34 & 0.19 & -0.57 & 0.27 & -0.206 & 0.032 & -0.365 & 0.106 & 17.181 & 11.652 & -0.153 & 0.02 & 122.1 & $1 \mathrm{P}$ \\
\hline 599 & 6.225902 & -72.84796 & 20.39 & 5000 & 98 & 2.7 & 0.2 & -0.51 & 0.20 & 0.03 & 0.27 & -0.272 & 0.047 & -0.361 & 0.088 & 23.915 & 7.979 & -0.172 & 0.02 & 115.3 & $1 \mathrm{P}$ \\
\hline 1517 & 6.292014 & -72.81255 & 20.42 & 4907 & 93 & 2.7 & 0.2 & -0.29 & 0.19 & 0.34 & 0.26 & 0.004 & 0.075 & -0.327 & 0.079 & 20.342 & 9.493 & -0.191 & 0.03 & 142.1 & $2 \mathrm{P}$ \\
\hline 1903 & 6.267869 & -72.80966 & 20.46 & 4980 & 97 & 2.7 & 0.2 & -0.73 & 0.20 & 0.48 & 0.26 & -0.056 & 0.041 & -0.474 & 0.052 & 20.352 & 9.258 & -0.166 & 0.03 & 141.9 & $2 \mathrm{P}$ \\
\hline 2349 & 6.115903 & -72.80645 & 20.15 & 4791 & 87 & 2.5 & 0.2 & -0.37 & 0.19 & -0.05 & 0.26 & -0.127 & 0.043 & -0.380 & 0.068 & 25.076 & 8.199 & -0.176 & 0.03 & 140.3 & $1 \mathrm{P}$ \\
\hline 2680 & 6.156927 & -72.80423 & 20.14 & 4814 & 88 & 2.5 & 0.2 & -0.35 & 0.21 & -0.79 & 0.28 & -0.102 & 0.063 & -0.375 & 0.063 & 22.207 & 9.929 & -0.176 & 0.03 & 141.6 & $1 \mathrm{P}$ \\
\hline 3130 & 6.231442 & -72.80103 & 19.91 & 4883 & 92 & 2.4 & 0.2 & -0.51 & 0.19 & 0.12 & 0.26 & -0.126 & 0.038 & -0.428 & 0.052 & 17.637 & 5.127 & -0.191 & 0.03 & 131.3 & $1 \mathrm{P}$ \\
\hline 3546 & 6.219912 & -72.79807 & 20.08 & 4747 & 85 & 2.4 & 0.2 & -0.59 & 0.21 & 0.70 & 0.27 & 0.091 & 0.088 & -0.412 & 0.072 & 21.487 & 6.405 & -0.153 & 0.03 & 130.2 & $2 \mathrm{P}$ \\
\hline 3910 & 6.219479 & -72.79566 & 19.98 & 4814 & 88 & 2.4 & 0.2 & -0.33 & 0.21 & 0.41 & 0.27 & 0.014 & 0.055 & -0.377 & 0.068 & 23.889 & 6.953 & -0.176 & 0.03 & 145.4 & $2 \mathrm{P}$ \\
\hline 5174 & 6.245548 & -72.78617 & 19.95 & 4791 & 87 & 2.4 & 0.2 & -0.52 & 0.21 & -0.10 & 0.27 & -0.143 & 0.037 & -0.385 & 0.057 & 22.241 & 5.330 & -0.174 & 0.03 & 141.2 & $1 \mathrm{P}$ \\
\hline 6130 & 6.175249 & -72.77926 & 20.18 & 4837 & 90 & 2.5 & 0.2 & -0.37 & 0.21 & -0.02 & 0.27 & -0.107 & 0.063 & -0.408 & 0.076 & 22.867 & 8.077 & -0.194 & 0.03 & 151.3 & $1 \mathrm{P}$ \\
\hline 6463 & 6.248931 & -72.77658 & 20.26 & 4791 & 87 & 2.5 & 0.2 & -0.24 & 0.21 & -0.49 & 0.28 & -0.156 & 0.048 & -0.391 & 0.072 & 20.158 & 8.311 & -0.173 & 0.03 & 148.2 & $1 \mathrm{P}$ \\
\hline 6862 & 6.206618 & -72.77339 & 19.90 & 4769 & 86 & 2.3 & 0.2 & -0.53 & 0.21 & 0.53 & 0.27 & 0.079 & 0.062 & -0.404 & 0.065 & 24.803 & 8.432 & -0.171 & 0.03 & 159.9 & $2 \mathrm{P}$ \\
\hline 7293 & 6.236802 & -72.76972 & 20.01 & 4683 & 82 & 2.3 & 0.2 & -0.41 & 0.21 & -0.29 & 0.27 & -0.153 & 0.058 & -0.373 & 0.059 & 27.081 & 9.327 & -0.177 & 0.03 & 141.2 & $1 \mathrm{P}$ \\
\hline 7963 & 6.302150 & -72.76238 & 20.04 & 4883 & 92 & 2.5 & 0.2 & -0.35 & 0.20 & -0.27 & 0.27 & -0.196 & 0.036 & -0.404 & 0.068 & 20.208 & 7.894 & -0.206 & 0.03 & 148.3 & $1 \mathrm{P}$ \\
\hline 220 & 6.271843 & -72.86605 & 21.18 & $\ldots$ & $\ldots$ & $\ldots$ & $\ldots$ & $\ldots$ & $\ldots$ & $\ldots$ & $\ldots$ & $\ldots$ & $\ldots$ & $\ldots$ & $\ldots$ & $\ldots$ & $\ldots$ & $\ldots$ & $\ldots$ & 145.8 & member \\
\hline 285 & 6.260201 & -72.86331 & 20.76 & $\ldots$ & $\ldots$ & $\ldots$ & $\ldots$ & $\ldots$ & $\ldots$ & $\ldots$ & $\ldots$ & $\ldots$ & $\ldots$ & $\ldots$ & $\ldots$ & $\ldots$ & $\ldots$ & $\ldots$ & $\ldots$ & 106.1 & member \\
\hline 791 & 6.207983 & -72.83820 & 19.72 & $\ldots$ & $\ldots$ & $\ldots$ & $\ldots$ & $\ldots$ & $\ldots$ & $\ldots$ & $\ldots$ & $\ldots$ & $\ldots$ & $\ldots$ & $\ldots$ & $\ldots$ & $\ldots$ & $\ldots$ & $\ldots$ & 107.4 & member \\
\hline 129 & 6.112807 & -72.87157 & 20.95 & $\ldots$ & $\ldots$ & $\ldots$ & $\ldots$ & $\ldots$ & $\ldots$ & $\ldots$ & $\ldots$ & $\ldots$ & $\ldots$ & $\ldots$ & $\ldots$ & $\ldots$ & $\ldots$ & $\ldots$ & $\ldots$ & 118.9 & field \\
\hline 176 & 6.293897 & -72.86896 & 20.57 & $\ldots$ & $\ldots$ & $\ldots$ & $\ldots$ & $\ldots$ & $\ldots$ & $\ldots$ & $\ldots$ & $\ldots$ & $\ldots$ & $\ldots$ & $\ldots$ & $\ldots$ & $\ldots$ & $\ldots$ & $\ldots$ & 130.9 & field \\
\hline 286 & 6.143601 & -72.87489 & 19.57 & $\ldots$ & $\ldots$ & $\ldots$ & $\ldots$ & $\ldots$ & $\ldots$ & $\ldots$ & $\ldots$ & $\ldots$ & $\ldots$ & $\ldots$ & $\ldots$ & $\ldots$ & $\ldots$ & $\ldots$ & $\ldots$ & 217.5 & field \\
\hline 329 & 6.066787 & -72.86083 & 20.76 & $\ldots$ & $\ldots$ & $\ldots$ & $\ldots$ & $\ldots$ & $\ldots$ & $\ldots$ & $\ldots$ & $\ldots$ & $\ldots$ & $\ldots$ & $\ldots$ & $\ldots$ & $\ldots$ & $\ldots$ & $\ldots$ & 89.5 & field \\
\hline 514 & 6.260530 & -72.82125 & 20.09 & $\ldots$ & $\ldots$ & $\ldots$ & $\ldots$ & $\ldots$ & $\ldots$ & $\ldots$ & $\ldots$ & $\ldots$ & $\ldots$ & $\ldots$ & $\ldots$ & $\ldots$ & $\ldots$ & $\ldots$ & $\ldots$ & 174.8 & field \\
\hline 651 & 6.227692 & -72.84573 & 19.91 & $\ldots$ & $\ldots$ & $\ldots$ & $\ldots$ & $\ldots$ & $\ldots$ & $\ldots$ & $\ldots$ & $\ldots$ & $\ldots$ & $\ldots$ & $\ldots$ & $\ldots$ & $\ldots$ & $\ldots$ & $\ldots$ & 90.6 & field \\
\hline 715 & 6.247569 & -72.84168 & 21.12 & $\ldots$ & $\ldots$ & $\ldots$ & $\ldots$ & $\ldots$ & $\ldots$ & $\ldots$ & $\ldots$ & $\ldots$ & $\ldots$ & $\ldots$ & $\ldots$ & $\ldots$ & $\ldots$ & $\ldots$ & $\ldots$ & 95.5 & field \\
\hline 825 & 6.213307 & -72.81841 & 20.57 & $\ldots$ & $\ldots$ & $\ldots$ & $\ldots$ & $\ldots$ & $\ldots$ & $\ldots$ & $\ldots$ & $\ldots$ & $\ldots$ & $\ldots$ & $\ldots$ & $\ldots$ & $\ldots$ & $\ldots$ & $\ldots$ & 112.0 & field \\
\hline 842 & 6.241302 & -72.83541 & 20.46 & $\ldots$ & $\ldots$ & $\ldots$ & $\ldots$ & $\ldots$ & $\ldots$ & $\ldots$ & $\ldots$ & $\ldots$ & $\ldots$ & $\ldots$ & $\ldots$ & $\ldots$ & $\ldots$ & $\ldots$ & $\ldots$ & 95.0 & field \\
\hline 905 & 6.257216 & -72.83270 & 20.73 & $\ldots$ & $\ldots$ & $\ldots$ & $\ldots$ & $\ldots$ & $\ldots$ & $\ldots$ & $\ldots$ & $\ldots$ & $\ldots$ & $\ldots$ & $\ldots$ & $\ldots$ & $\ldots$ & $\ldots$ & $\ldots$ & 152.2 & field \\
\hline 1201 & 6.194623 & -72.81501 & 20.83 & $\ldots$ & $\ldots$ & $\ldots$ & $\ldots$ & $\ldots$ & $\ldots$ & $\ldots$ & $\ldots$ & $\ldots$ & $\ldots$ & $\ldots$ & $\ldots$ & $\ldots$ & $\ldots$ & $\ldots$ & $\ldots$ & 94.3 & field \\
\hline 4281 & 6.236492 & -72.79306 & 20.66 & $\ldots$ & $\ldots$ & $\ldots$ & $\ldots$ & $\ldots$ & $\ldots$ & $\ldots$ & $\ldots$ & $\ldots$ & $\ldots$ & $\ldots$ & $\ldots$ & $\ldots$ & $\ldots$ & $\ldots$ & $\ldots$ & 94.8 & field \\
\hline 4542 & 6.272315 & -72.79084 & 20.11 & $\ldots$ & $\ldots$ & $\ldots$ & $\ldots$ & $\ldots$ & $\ldots$ & $\ldots$ & $\ldots$ & $\ldots$ & $\ldots$ & $\ldots$ & $\ldots$ & $\ldots$ & $\ldots$ & $\ldots$ & $\ldots$ & 176.8 & field \\
\hline 4873 & 6.270678 & -72.78851 & 20.92 & $\ldots$ & $\ldots$ & $\ldots$ & $\ldots$ & $\ldots$ & $\ldots$ & $\ldots$ & $\ldots$ & $\ldots$ & $\ldots$ & $\ldots$ & $\ldots$ & $\ldots$ & $\ldots$ & $\ldots$ & $\ldots$ & 138.4 & field \\
\hline 5697 & 6.220817 & -72.78246 & 20.28 & $\ldots$ & $\ldots$ & $\ldots$ & $\ldots$ & $\ldots$ & $\ldots$ & $\ldots$ & $\ldots$ & $\ldots$ & $\ldots$ & $\ldots$ & $\ldots$ & $\ldots$ & $\ldots$ & $\ldots$ & $\ldots$ & 213.6 & field \\
\hline 7604 & 6.231820 & -72.76645 & 20.23 & $\ldots$ & $\ldots$ & $\ldots$ & $\ldots$ & $\ldots$ & $\ldots$ & $\ldots$ & $\ldots$ & $\ldots$ & $\ldots$ & $\ldots$ & $\ldots$ & $\ldots$ & $\ldots$ & $\ldots$ & $\ldots$ & 166.7 & field \\
\hline
\end{tabular}

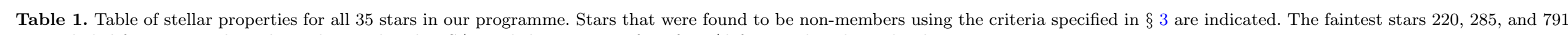
are excluded from our analysis due either to their low $\mathrm{S} / \mathrm{N}$ and the presence of artifacts/defects within the molecular spectra. 